\title{
Chipless RF Liquid Sensor
}

\author{
A.J.Cole, C.C.H.Ng, F.A.Almalki, P.R.Young \\ School of Engineering and Digital Arts \\ University of Kent \\ Canterbury, United Kingdom \\ ajc87@kent.ac.uk
}

\begin{abstract}
A method for the wireless evaluation of the dielectric permittivity of liquids is presented. The use of a thinfilm, slotted waveguide sleeve around a standard unplasticised polyvinyl chloride pipe is proposed. Wireless interrogation of the slot with a plane wave excites a resonant mode whose frequency is dependent on the dielectric permittivity within the pipe. The proposed method allows for measurements to be taken in situ with no need for samples. The device has good potential for very low-cost monitoring applications, which may require real-time, continuous assessment.
\end{abstract}

Keywords-Waveguide; dielectric measurements; microwave sensors; chipless.

\section{INTRODUCTION}

There are many different applications of microwave remote sensing [1], whether active or passive. Microwave sensors have been used to monitor or measure in a variety of scenarios [2-6]. In industrial processes where pipes are commonly used to distribute liquids, it would be beneficial to have a low-cost, wireless system able to monitor their contents. It is possible to fabricate devices whose resonance is altered by the presence of media with different dielectric constants. By utilising industrial liquids as such media, known permittivities can be exploited to provide a family of resonances, which can be used to determine the particular liquid present.

In [7], Lobato-Morales et al. make use of the cavity perturbation technique for the assessment of dielectric properties. A rectangular waveguide containing a narrow height epsilon-near-zero (ENZ) channel operating at 5.4-GHz is shifted by $660-\mathrm{MHz}$ by samples placed in the centre of the ENZ tunnel. As an extension to this work [8], Lobato-Morales et al. implemented a wireless sensor to measure the complex dielectric permittivity of industrial liquids using passive tag antennas connected to a substrate-integrated-waveguide (SIW). The tag operates at $4 \mathrm{GHz}$ and is capable of measuring small sample volumes with dielectric constants below 20. In [9], Verma et al. utilise the Q-factor of a microstrip patch to determine the complex permittivity of various dielectrics.

This paper outlines the potential application of thin-film technology to create a chipless RF sensor to measure the dielectric properties of liquids inside a length of pipe. The possibility for in situ wireless measurements of liquids flowing within a nonmetal pipe could have strong potential in industrial processes.

The paper layout is as follows. Section II outlines the wireless sensing method, section III characterises the sensor, and section IV presents the liquid sensing results.

\section{LIQUID SENSING METHOD}

Circular waveguide provides useful geometry for liquid sensing, as the use of thin films such as Mylar allows for the fabrication of copper sleeves which can be wrapped around pipes of varying radii. By inserting a slot along the length of the waveguide and essentially creating an elongated split-ring resonator (SRR), it is possible to excite the device with an incident plane wave. Fig. 1 displays the electric field distribution across the radial cross-section of an excited, unplasticised polyvinyl chloride (uPVC) pipe fitted with an external slotted waveguide sleeve. The field is a maximum at the slot, and reduces to zero at the opposite boundary, where a significant portion exists within the space inside the uPVC pipe walls. At resonance, the field encounters an inhomogeneous medium consisting of two dielectric layers, the uPVC pipe and its internal contents. Assuming constant dimensions, the resonant frequency of the slot depends on the permittivity of these contents, and thus can be used to determine the electrical properties of liquids flowing within the pipe.

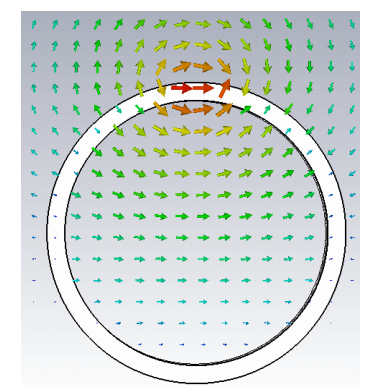

Fig. 1. Simulated electric field at resonance 


\section{DEVICE CHARACTERISATION}

With no liquid(s) present, the slot resonance is determined by several parameters. The simulated frequency response of the sensor is assessed by observing the reflection of an incident plane wave orientated in the $x$-direction. For all simulations (for unchanged parameters) the slot width and length is $2 \mathrm{~mm}$ and $50 \mathrm{~mm}$ respectively, and uPVC pipe thickness is $1.2 \mathrm{~mm}$. The electrical properties of uPVC are difficult to find in literature, but a survey of various specifications returns a dielectric constant $\varepsilon_{r}=3$. Fig. 2 displays the geometry of the sensor, the slot length is aligned in the $y$-direction. All simulations were performed in Computer Simulation Technology's (CST) Microwave Studio and neglect uPVC loss.

Fig. 3 shows the effect of changing the outer radius of the uPVC pipe, varying from $6 \mathrm{~mm}$ to $14 \mathrm{~mm}$. The sensor behaves similarly to the single split ring detailed in [10], and in fact when the uPVC is replaced by air, the simulated resonant frequency is $1.77-\mathrm{GHz}$, which matches the numerical result of a $10 \mathrm{~mm}$ radius single split ring in [10]. Fig. 4 displays the lesser effect of varying the slot length, where a change in almost 50mm has a significant but reduced impact. A longer slot length does, however, provide a larger effective apperture which will increase the magnitude of the reflected signal. Finally, Fig. 5 shows the effect of changing the slot width. It can be seen that the device continues to operate with very small dimensions, although a width of $2 \mathrm{~mm}$ has been used for liquid measurements.

\section{LIQUID SENSING}

Three industrial chemicals have been assessed: Butanol, Ethanol and Methanol. The electrical properties of each liquid were obtained from [11], where Gregory and Clarke present various models for the complex permittivities of each liquid. The dispersion data was imported into CST, where all liquids are assumed to be slightly below room temperature, at 20C.

Fig. 6 shows the S11 results for air, Butanol and Ethanol. The uPVC loss is neglected, explaining the peak for an airfilled pipe, whereas Butanol and Ethanol have relatively high loss tangents, as seen in Fig. 7, and therefore attenuate at resonance. Methanol has a very high dielectric constant at low frequencies, and the majority of the electric field is contained within the walls of the pipe, rather than the liquid. For a reasonable result it would be necessary to operate at higher frequencies where the dielectric constant is lower, as can be seen in Fig. 8. It is also interesting to note that Methanol has a

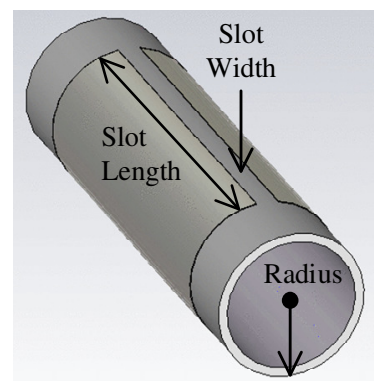

Fig. 2. Simulated liquid sensor

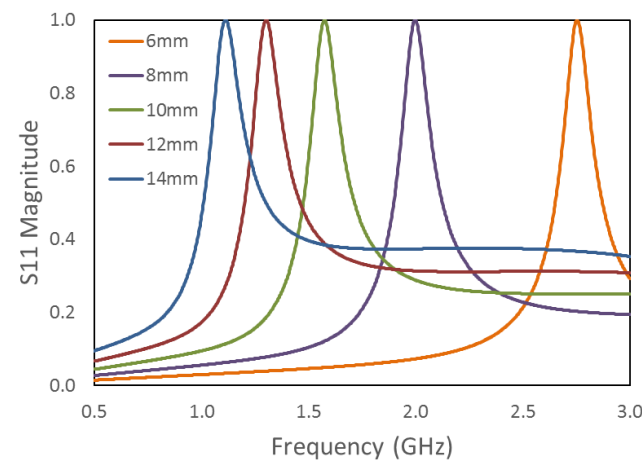

Fig. 3. Simulated frequency response for different radii

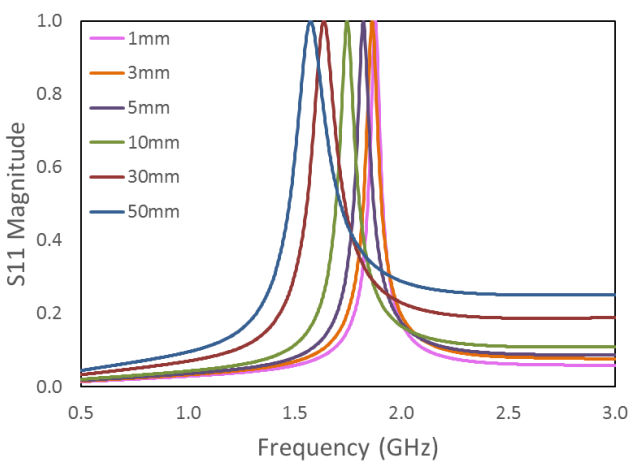

Fig. 4. Simulated frequency response for different slot lengths

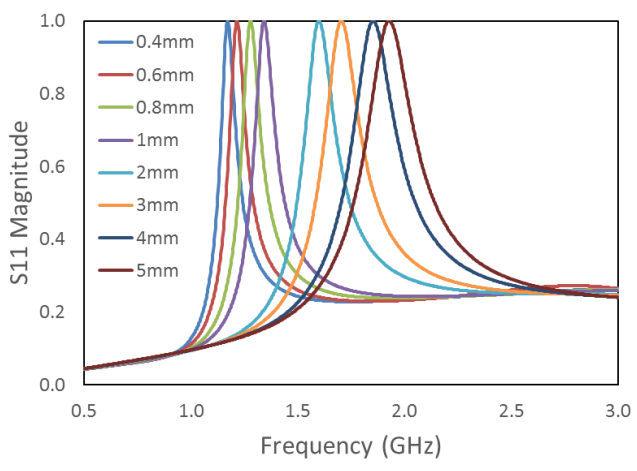

Fig. 5. Simulated frequency response for different slot widths

considerably lower loss tangent than Butanol and Ethanol, shown in Fig. 7, and this could perhaps contribute to lack of any significant attenuation, as observed with the other liquids.

As a caveat of using a longer slot length to provide a larger effective aperture, it is possible to detect variations in the level of liquid. Assuming a vertically placed pipe, where liquid moves up or down, the frequency response should shift between an air-filled pipe when empty, and a liquid-filled pipe when full. Indeed, Fig. 9 shows the effect of emptying a Butanol-filled pipe in $5 \mathrm{~mm}$ increments. Changing the level of ethanol yielded similar results.

The permittivity of each liquid is temperature dependent [11], particularly at lower frequencies. Fig. 10 shows the effect of temperature on the dielectric constant of Ethanol. Given the changes in value, the sensor would be capable of detecting relatively small variations in temperature. 


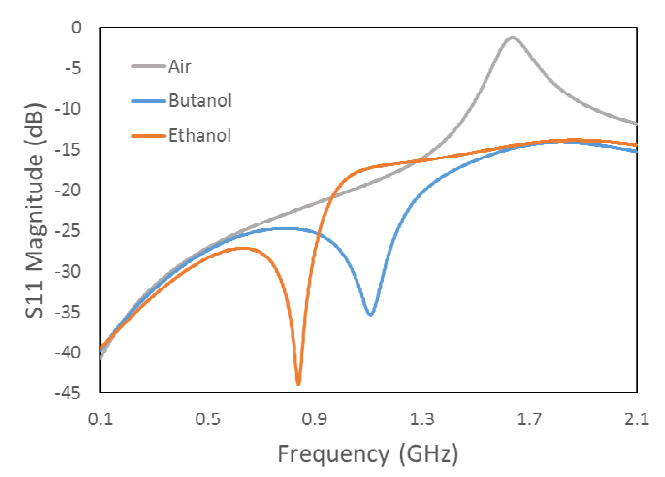

Fig. 6. Simulated S11 magnitudes for air, Butanol and Methanol

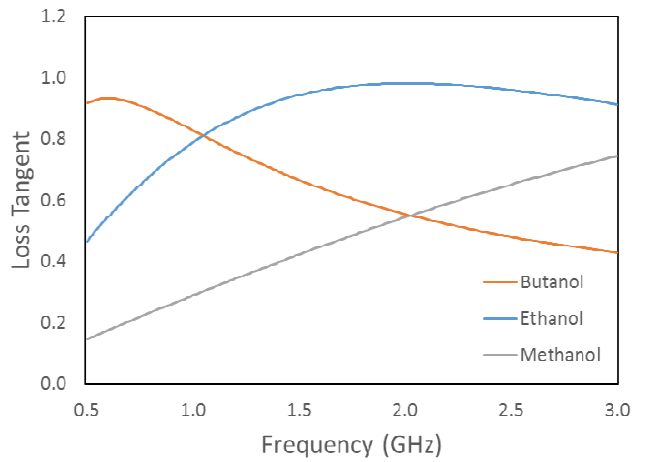

Fig. 7. Calculated loss tangent for Butanol, Ethanol and Methanol

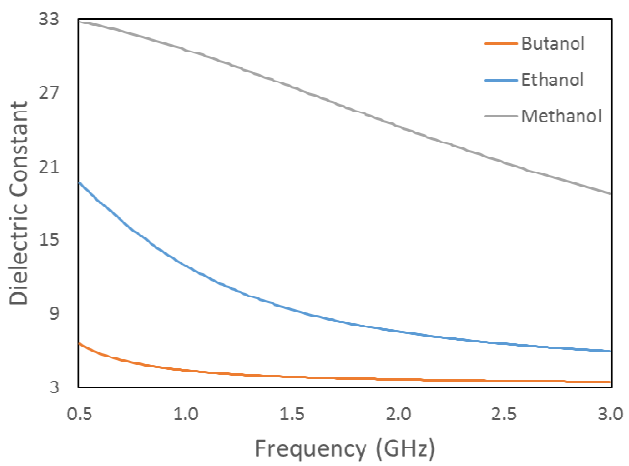

Fig. 8. Calculated dielectric constant for Butanol, Ethanol and Methanol

\section{CONCLUSION}

A very low-cost, chipless RF sensor for the assessment of the electrical properties of liquids within UPVC pipe has been presented. The sensor makes use of the geometry of circular waveguide and the availability of thin-film substrates. Simulated results show good operation with industrial liquids, as well as the possibility for measurements of level and temperature. The tolerance of slot length allows for a range of device sizes, and the possibility to vary the frequency response through changing slot width and pipe radius provides flexibility. Liquid measurements are to be taken and will subsequently be presented in a full paper.

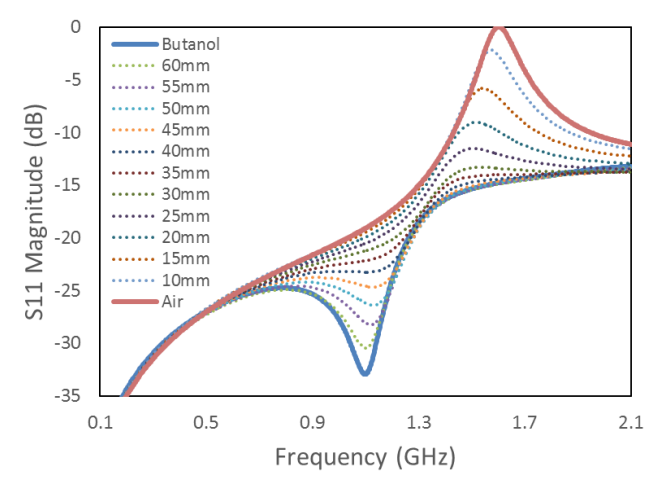

Fig. 9. Simulated frequency response of a changing Butanol level

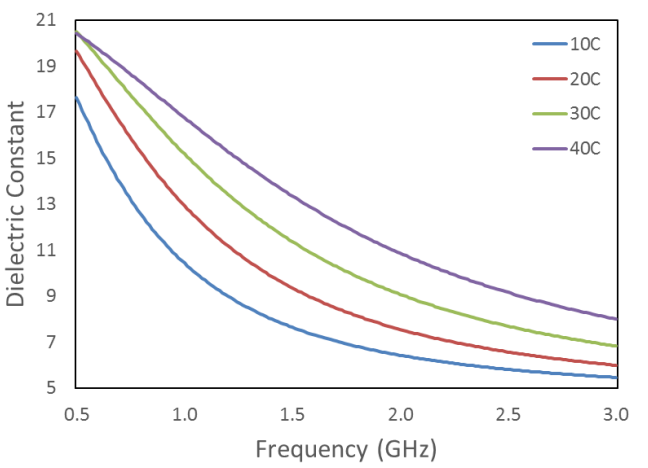

Fig. 10. Effect of temperature on the dielectric constant of Ethanol

\section{REFERENCES}

[1] Canada Centre for Remote Sensing, Fundamentals of Remote Sensing, Natural Resources Canada, Chapter 1, pp. 5 - 19.

[2] Canada Centre for Remote Sensing, Fundamentals of Remote Sensing, Natural Resources Canada, Chapter 5, pp. 174 - 239.

[3] Tsenchien Chiu, "Dielectric constant measurement technique for a dielectric strip using a rectangular waveguide," IEEE Trans. Instrum. Meas., Vol. 52, No. 5, Oct 2003.

[4] V. Sekar, W. J. Torke, S. Palermo and K. Entesari, "A self-sustained microwave system for dielectric constant measurement of lossy organic liquids," IEEE Trans. Microw. Theory Tech., Vol. 60, No. 5, May 2012.

[5] T. Kobayashi and Y. Nikawa, "Dynamic measurement of temperature dependent permittivity of liquid material using microwaves," in Proc. Asia-pacific Macrow. Conf., Yokohama, Japan, Dec. 2010, pp. 13681371.

[6] S. Seewattanapon, T. Wattakeekamthorn, T. Somwong and P. Akkaraekthalin, "A microstrip folded resonator sensor for measurement of dielectric constant," in Proc. ECTI-CON 2008, pp. 245-248.

[7] H. Lobato-Morales and A. Corona-Chavez, "Permittivity measurements at Microwave frequencies using Epsilon-Near-Zero (ENZ) tunnel structure,” IEEE Trans. Micros. Theory Tech., Vol. 59, No. 7, July 2011.

[8] H. Lobato-Morales and A. Corona-Chavez, "Wireless sensing of complex dielectric permittivity of liquids based on the RFID," IEEE Trans. Microw. Theory Tech., Vol. 62, No. 9, Sept 2014.

[9] A. Verma, Nasimuddin and A. Omar, 'Microstrip resonator sensors for determination of complex permittivity of materials in sheet, liquid and paste forms', IEE Proceedings - Microwaves, Antennas and Propagation, vol. 152, no. 1, p. 47, 2005.

[10] A. Radkovskaya et al., 'Resonant frequencies of a combination of split rings: Experimental, analytical and numerical study', Microwave and Optical Technology Letters, vol. 46, no. 5, pp. 473-476, 2005.

[11] A. P, Gregory and R. N. Clarke, "Tables of the complex permittivity of dielectric reference liquids up to $5 \mathrm{GHz}$," CETM, Teddington, U.K., Nat. Phys. Rep. 33, Jan 2012, pp. 19-61. 